\title{
BDRG AND SHOK INSTRUMENTS FOR STUDY OF GRB PROMPT EMISSION IN MICHAYLO LOMONOSOV SPACE MISSION
}

\author{
A.M. Amelushkin ${ }^{1}$, V.V. Bogomolov ${ }^{1}$, V.I. Galkin ${ }^{1}$, B.V. Goncharov ${ }^{1}$, \\ E.S. Gorbovskoy ${ }^{2}$, V.G. Kornilov ${ }^{2}$, V.M. Lipunov ${ }^{2}$, M.I. Panasyuk ${ }^{1}$, \\ V.L. Petrov ${ }^{1}$, G.F. Smoot $^{3}$, S.I. Svertilov ${ }^{1}$, N.N. Vedenkin ${ }^{1}$ \\ and I.V. Yashin ${ }^{1}$
}

\begin{abstract}
The study of GRB prompt emission (PE) is one of the main goals of the Lomonosov space mission, which is being prepared at Moscow State University. The GRB monitor (BDRG) and the widefield optical cameras (SHOK) are intended for detection of GRB prompt emission as well as optical counterparts. The BDRG instrument consists of three identical $\mathrm{NaI}(\mathrm{Tl}) / \mathrm{CsI}(\mathrm{Tl})(13.0 \times 2.0 \mathrm{~cm} \varnothing)$ phoswich detectors, whose axes determine the Cartesian coordinate system. This allows to localize any GRB source on the sky by means of the count rate seen by each detector with an accuracy of $\sim 2 \mathrm{deg}$. The SHOK instrument consists of two identical wide-field cameras (WFC) directed in such a way that the field of view (FOV) of each WFC overlaps by the corresponding BDRG FOV, which produces a trigger on the WFC in case of a GRB detection. With this setup, the GRB prompt light curve will be obtained in the visible without any delay with respect to gamma-rays, which is crucial for a GRB central engine understanding.
\end{abstract}

\section{Introduction}

GRBs are one of the most energetic phenomena in the Universe. They appear as short (from dozens of milliseconds up to dozens of seconds) increases of fluxes of gamma radiation with typical energy of tens or hundreds of $\mathrm{keV}$ and sometimes are

\footnotetext{
${ }^{1}$ D.V. Skobeltsyn Institute of Nuclear Physics of M.V. Lomonosov Moscow State University, Leninskie Gory, 1/2, 119991 Moscow, Russia

${ }^{2}$ P.K. Shternberg Astronomical Institute of M.V. Lomonosov Moscow State University,

Universitetskii prosp. 17, 119992 Moscow, Russia

${ }^{3}$ Berkeley Center for Cosmological Physics, Berkeley, California, USA
} 
observed in hard gamma-rays with energy of at least $10^{9} \mathrm{eV}$. Over the last years, $\mathrm{UV} /$ optical telescopes follow-ups have allow to perform multiwavelength studies of afterglows (Gehrels et al. 2009).

Usually UV and optical observations start at several tens of seconds after the beginning of the burst in gamma-rays. This delay is caused by the delayed trigger as well as for the repointing of optical instrument towards the given GRB direction Thus the optical radiation appearing during the first stages of the bursts (optical prompt emission) is not well studied yet.

The Lomonosov scientific program includes a multi-wavelength GRB study at different epochs (Sadovnichii et al. 2012). In particular, prompt emission measurements will be provided based on the direct observations by the wide field optical cameras and other instruments onboard Lomonosov, namely: a gammaray spectrometer (BDRG) and a wide field optical camera (SHOK) (which will be presented in this manuscript) besides a gamma-ray coded-mask detector and a fast reaction UV/optical telescope (UFFO-p, described elsewhere).

\section{Design and characteristics of BDRG gamma-ray spectrometer}

The BDRG gamma-ray spectrometer for the Lomonosov mission is designed in order to obtain temporal and spectral information about GRBs in energy range 10-3000 keV as well as to produce GRB trigger for the other instruments and missions.

The BDRG instrument consists of 3 similar detector boxes, connected to the data analysis box (see Fig. 1). The BDRG detectors axes are shifted $90^{\circ}$ from each other. Detectors have cosine angular dependence of sensitive area (FWHM $\sim 60^{\circ}$ ) that allows one to determine the coordinates of GRB with accuracy of few degrees for bright GRBs by comparing the different detector countrates. The instrument is sensitivite to bursts with fluences down to $10^{-7} \mathrm{erg} / \mathrm{cm}^{2}$. Each detector consists of optically coupled thin $(3 \mathrm{~mm}) \mathrm{NaI}(\mathrm{Tl})$ and considerably thick $(17 \mathrm{~mm})$ $\mathrm{CsI}(\mathrm{Tl})$ crystals. The thickness of $\mathrm{NaI}(\mathrm{Tl})$ is optimized for soft part of energy range. $\mathrm{CsI}(\mathrm{Tl})$ plays a role of active shield for soft radiation being the main detector for harder energy photons. Working ranges are $0.01-0.5 \mathrm{MeV}$ for $\mathrm{NaI}(\mathrm{Tl})$ and $0.05-3 \mathrm{MeV}$ for $\mathrm{CsI}(\mathrm{Tl})$ one. Detector boxes have mass $\sim 5.5 \mathrm{~kg}$ and power consumption $<3 \mathrm{~W}(\sim 15 \mathrm{~W}$ for the data analysis box $)$.

\subsection{Detector box electronics design and calibration principles}

A pulse of current originates from the corresponding photomultiplier (PMT) leading to the amplitude discriminator generating an "event start" pulse. A sequence of control signals formed after the pulse is used for the change of "SPDT state" so the two parts of the primary PMT output pulse are integrated independently. The first one is proportional to the amount of light collected during the first $800 \mathrm{~ns}$ of the pulse (so called "Fast component") and the second one is proportional to the amount of light collected during the next $2 \mu \mathrm{s}$ (the so called "Slow component"). Both fast and slow components are analyzed at the BDRG information box 


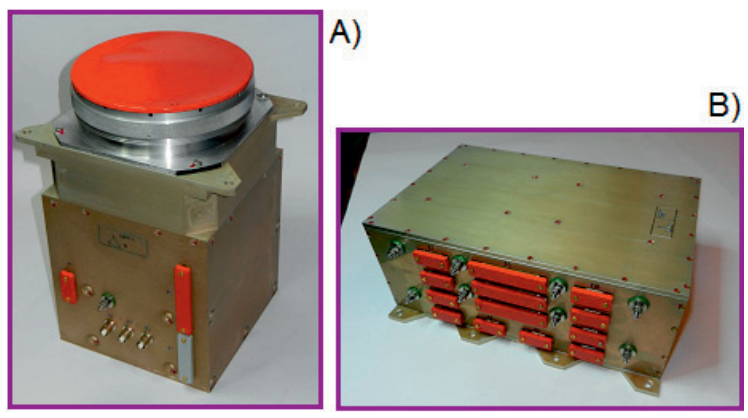

Fig. 1. The BDRG instrument: A) The detector box. B) The data analysis box.

(BA BDRG) in order to determine in which scintillator the interaction took place and the value of energy release. A sequence of output data frames is produced in the BA BDRG box allotting for the analysis of the GRB data.

The calibration procedure used for BDRG instrument can be explained using 2D-diagram where $\mathrm{x}$ and $\mathrm{y}$ values for each event represents its fast and slow component amplitudes. An example of such diagram for ${ }^{137} \mathrm{Cs}$ gamma-source is presented in Figure 2. One can see two straight lines for $\mathrm{NaI}(\mathrm{Tl})$ and $\mathrm{CsI}(\mathrm{Tl})$ events. The points between these lines correspond to the events when Compton interaction of a gamma-quantum took place so the energy was released in both crystals. The spots of events with energy release $662 \mathrm{keV}$ correspondent to total energy absorption peak of ${ }^{137} \mathrm{Cs}$ source radiation as well as $1.46 \mathrm{MeV}$ and $2.614 \mathrm{MeV}$ events from background isotopes ${ }^{40} \mathrm{~K}$ and ${ }^{208} \mathrm{Tl}$ (from ${ }^{232} \mathrm{Th}$ decay sequence) are seen.

One can see a spot of $32 \mathrm{keV}$ X-rays on the detailed subpicture. These events are present only in $\mathrm{NaI}(\mathrm{Tl})$ part of detector because most of such X-rays interact with it and do not get to $\mathrm{CsI}(\mathrm{Tl})$ layer. A red cross shows a zero point correspondent to zero amplitude of the PMT pulse. This point is shifted from $(0,0)$ by some small constant voltage level at ADC inputs. The energy release can be determined from the distance between the point of event and this zero point individually for each scintillator. One can see that events in $\mathrm{NaI}(\mathrm{Tl})$ and $\mathrm{CsI}(\mathrm{Tl})$ are well separated by an energy release greater than $10 \mathrm{keV}$.

\subsection{Production of BDRG trigger}

The BDRG monitoring readings demonstrate a fast increase of gamma-ray flux if the trigger is produced. Necessary conditions for trigger production are:

- Presence of fast rise of hard X-rays readings (channel 25-100 keV will be used in BDRG).

- Not too high rate in hard X-rays.

- GRB/imitation by electrons criterion based on $\mathrm{NaI}(\mathrm{Tl}) / \mathrm{CsI}(\mathrm{Tl})$ ratio (see below). 


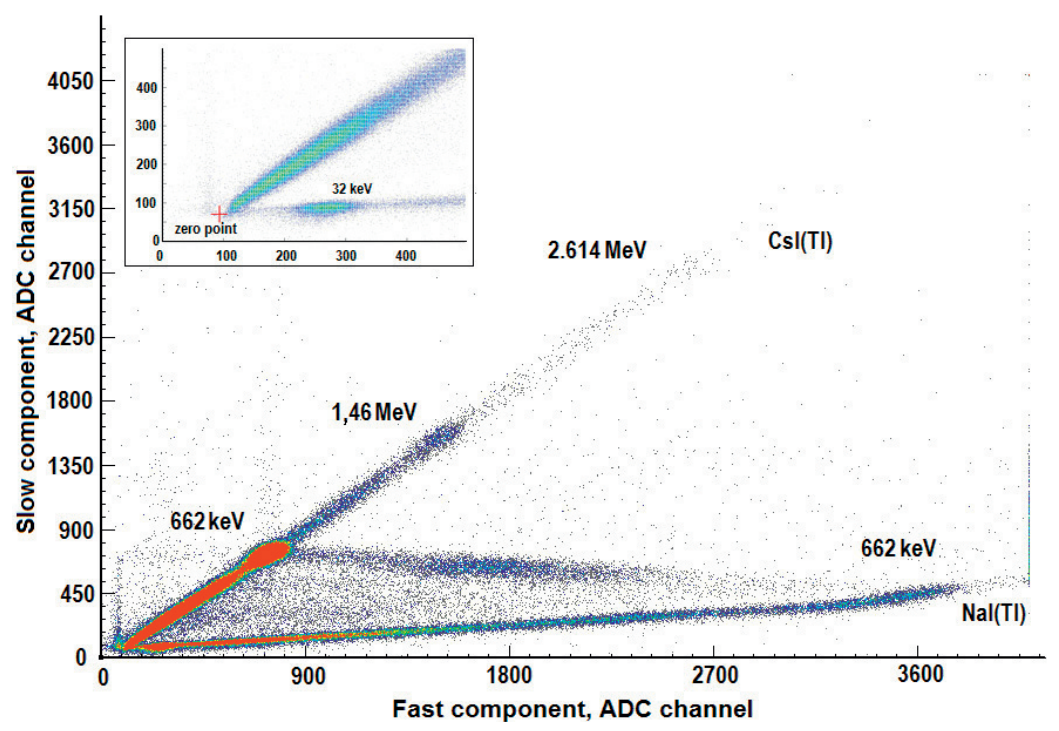

Fig. 2. Example of $2 \mathrm{D}$-diagram (slow part of PMT pulse vs. fast one) for ${ }^{137} \mathrm{Cs}$ gammarays.

The algorithm for detecting a fast rise in the gamma-ray countrates is illustrated in Figure 3. Countrates are stored in memory with temporal resolution $\Delta \mathrm{T}$ (temporal resolution of burst data to be transmitted to Earth) for $100 * \Delta \mathrm{T}$ interval. The track record (the recording history) is divided into $5^{*} \Delta \mathrm{T}$ bins (row $\mathrm{N}_{5 \mathrm{i}}$ ) with the sums $\mathrm{N}_{100}$ and $\mathrm{N}_{50}$ being the numbers of events for the first and the second half of countrate history, which are properly determined (see Fig. 3). By means of linear regression, the expected value for the next $5^{*} \Delta \mathrm{T}$ interval is calculated (named $\mathrm{N}_{5}$ exp). If $\mathrm{N}_{50}<30$, the mean value is used. Then, the standard deviation $\sigma$ is calculated for the difference between the value of $\mathrm{N}_{5 \mathrm{i}}$ and the one obtained by the regression method. Then the values $\mathrm{N}_{5}$ and $\mathrm{N}_{5 \exp }$ are compared each other. Thus, a trigger is produced if:

$$
\left\{\begin{array}{l}
\mathrm{N}_{5}>\mathrm{N}_{5 \exp }+\mathrm{N} * \sigma \\
\mathrm{N}_{5}>2
\end{array}\right.
$$

$\mathrm{N}$ is the number of standard deviations when the increase is considered to be significant.

Several timescales will be used for independent triggering: $20 \mathrm{~ms}, 1 \mathrm{~s}$ and $20 \mathrm{~s}$. The threshold (value $\mathrm{N}$ in formula above) can be chosen by analysis of empiric distribution of triggers. An example of such distribution for $20 \mathrm{~ms}$ and $1 \mathrm{~s}$ triggering is shown in Figure 4. The criterion for onboard triggering can be soft allowing one to have $\sim 20$ false bursts per day transferred to Earth but not to loose any real GRB with enough counrate. For the presented case it is set to $\mathrm{N}_{\text {sigm }}>9$ for $20 \mathrm{~ms}$. The criterion used for the alerts distributed to world net must be harder. Proposed value is $\mathrm{N}_{\text {sigm }}>12$ or even greater. 


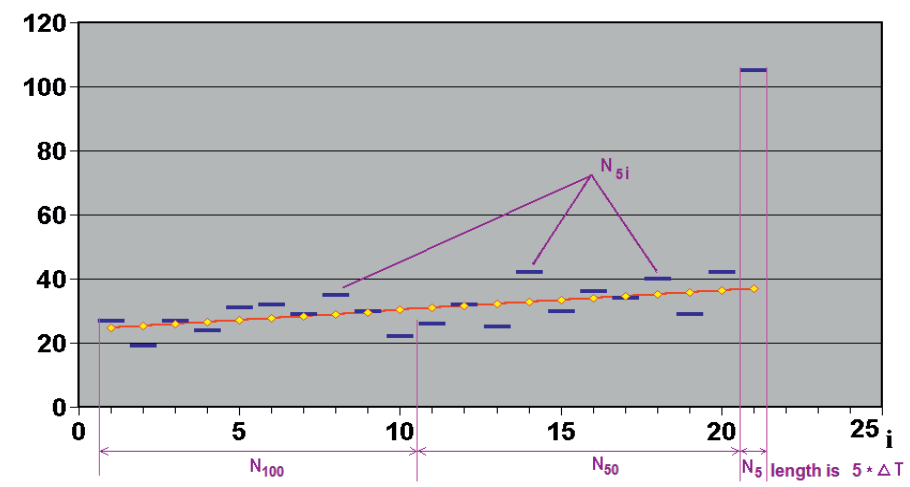

Fig. 3. Illustration for the algorithm of search for fast rise of gamma-ray countrates.
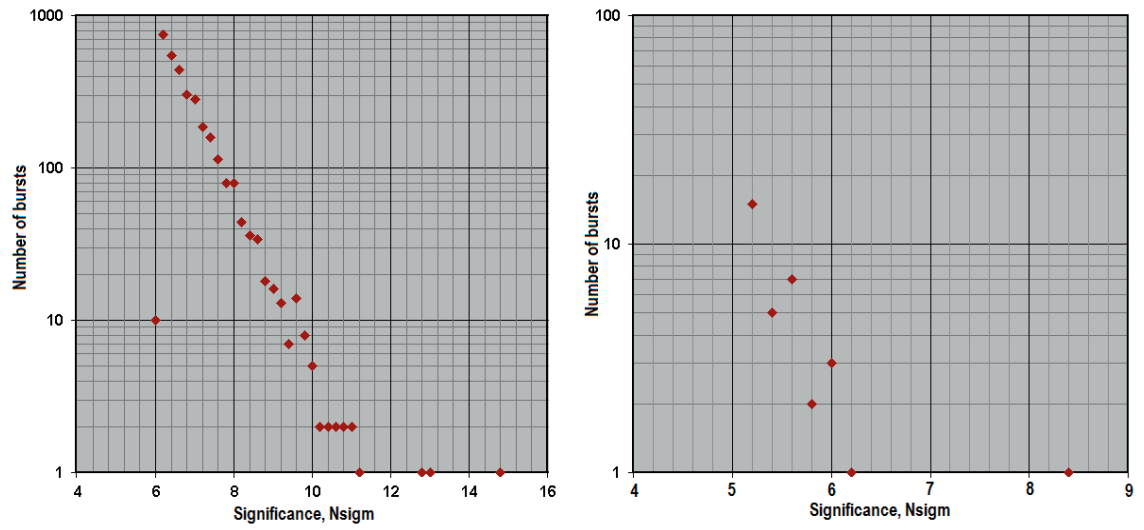

Fig. 4. Distribution of $20 \mathrm{~ms}$ (left) and $1 \mathrm{~s}$ (right) triggers from a $65 \mathrm{~h}$ background measurement.

It is necessary to be sure that the fast rise of BDRG readings was caused by true GRB gamma-rays and not by bremsstrahlung x-rays coming from the satellite when it some particles beam passes thru. The criterion used in BDRG is based on the comparison of countrates seen by $\mathrm{NaI}(\mathrm{Tl})$ and $\mathrm{CsI}(\mathrm{Tl})$ in different energy channels. The parameters of the criterion such as the limits of energy intervals and $\mathrm{NaI}(\mathrm{Tl}) / \mathrm{CsI}(\mathrm{Tl})$ threshold ratio were chosen by modelling using the Geant software. They can be also changed by commands during the space experiment.

\subsection{Estimation of GRB coordinates}

The GRB position can be estimated by comparing the countrates recorded at the 3 BDRG detectors due the having cosine (incident angle) effect. The 3 detectors are tilted $90^{\circ}$ from each other so the GRB direction can be calculated from the formula $\cos \theta_{\mathrm{i}}=\mathrm{N}_{\mathrm{i}} / \operatorname{sqrt}\left(\mathrm{N}_{1}^{2}+\mathrm{N}_{2}^{2}+\mathrm{N}_{3}^{2}\right)$ where $\theta_{\mathrm{i}}$ is the angle between the detector 
axe and the burst direction and $\mathrm{N}_{\mathrm{i}}$ is the number of events in the detector number i. The accuracy of GRB localization depends on GRB brightness, hardness and background level. The results of numeric modelling and preliminary laboratory tests with ${ }^{241} \mathrm{Am}$ radioactive source are presented in Figure 5.

\begin{tabular}{|c|c|c|c|c|c|c|c|}
\hline \multirow{2}{*}{ fluence } & \multirow{2}{*}{$\mathrm{kT}, \mathrm{keV}$} & \multicolumn{7}{|c|}{ Background rate, $\mathrm{Hz}$} \\
\cline { 3 - 8 } & & 0 & 10 & 50 & 100 & 500 & 1000 \\
\hline \hline $1.0 \mathrm{E}-07$ & 5 & 18.5 & 27 & 36 & 40 & 48 & 52 \\
\hline $1.0 \mathrm{E}-07$ & 10 & 8.4 & 10.3 & 16.5 & 21 & 33 & 38 \\
\hline $1.0 \mathrm{E}-07$ & 25 & 5.2 & 6 & 9.5 & 12 & 21 & 27 \\
\hline $1.0 \mathrm{E}-07$ & 50 & 5 & 5.6 & 9 & 11 & 19.2 & 25 \\
\hline $1.0 \mathrm{E}-07$ & 100 & 5.5 & 7 & 11 & 14 & 24 & 28 \\
\hline $1.0 \mathrm{E}-07$ & 300 & 8.5 & 11.5 & 19 & 24 & 37 & 43 \\
\hline $1.0 \mathrm{E}-06$ & 5 & 8 & 8.5 & 10.5 & 13 & 21 & 26 \\
\hline $1.0 \mathrm{E}-06$ & 10 & 4.5 & 4.5 & 5 & 5.5 & 8 & 10 \\
\hline $1.0 \mathrm{E}-06$ & 25 & 2.2 & 2.2 & 2.5 & 2.8 & 4.5 & 5.2 \\
\hline $1.0 \mathrm{E}-06$ & 50 & 2 & 2 & 2.4 & 2.7 & 4.5 & 5.2 \\
\hline $1.0 \mathrm{E}-06$ & 100 & 2.4 & 2.5 & 2.8 & 3.4 & 5.2 & 6.3 \\
\hline $1.0 \mathrm{E}-06$ & 300 & 3.7 & 4.1 & 4.5 & 5.2 & 8.3 & 10.5 \\
\hline \hline $1.0 \mathrm{E}-05$ & 5 & 7 & 7 & 7 & 7 & 7 & 8 \\
\hline $1.0 \mathrm{E}-05$ & 10 & 4.5 & 4.5 & 4.5 & 4.5 & 4.5 & 4.5 \\
\hline $1.0 \mathrm{E}-05$ & 25 & 1.8 & 1.8 & 1.8 & 1.8 & 1.8 & 1.8 \\
\hline $1.0 \mathrm{E}-05$ & 50 & 1.6 & 1.6 & 1.6 & 1.6 & 1.6 & 1.8 \\
\hline $1.0 \mathrm{E}-05$ & 100 & 2.2 & 2.2 & 2.2 & 2.2 & 2.4 & 2.4 \\
\hline $1.0 \mathrm{E}-05$ & 300 & 3 & 3 & 3 & 3 & 3 & 3.5 \\
\hline
\end{tabular}
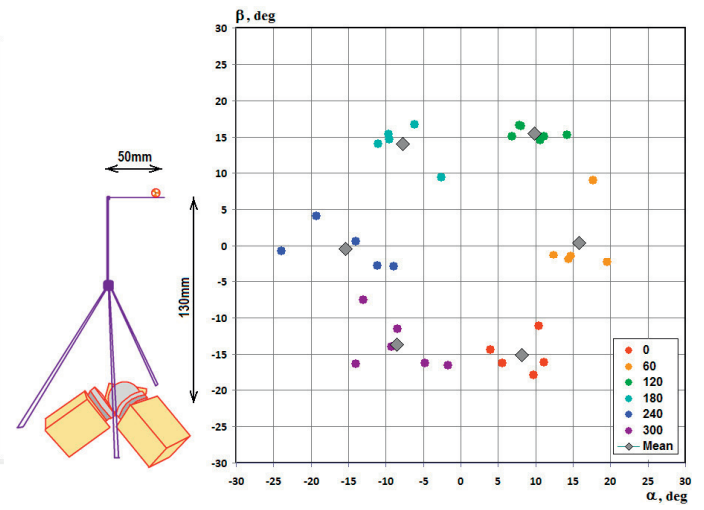

Fig. 5. Left: accuracy of GRB positioning (degrees) estimated by numeric simulations. Right: result of preliminary laboratory tests with ${ }^{241} \mathrm{Am}$ radioactive source as shown in the sketch. Depending on the distance of the source to the main axis ( 0 for on-axis, $60 \mathrm{~mm}, 120 \mathrm{~mm}$, etc.) the localization in the $(\alpha, \beta)$ plane varies significantly.

\section{SHOK wide field optical camera}

The SHOCK instrument consists of two stationary wide-angle fast cameras. Their field of view is situated within the area of gamma-bursts' detection of other instruments onboard the "Lomonosov" satellite (see simplified FOV together with SHOK photo in Fig. 6). Each SHOK unit is an optical camera with a wide FOV about $20^{\circ} \times 40^{\circ}$, and maximum framing rate about 5-7 frames/sec with sensitivity $\sim 11$ mag. In fact, cameras record "a movie" continuously, and in case of gamma-ray burst detection part of this movie can be fixed and transmitted to the Earth. The amount of data is $\sim 700 \mathrm{Mb}$ /burst. GRB light curves will be fixed in the optical and gamma-ray ranges simultaneously, and a significant opportunity for measuring of optical curves of the gamma-ray burst prompt emission and their precursors' detecting are provided.

\section{Conclusion}

Expected numbers of GRBs were estimated taking into account the instruments FOV as well as the background conditions on low altitude orbit. Based on previous missions, one can expect $~ 150$ GRB triggers per year from BDRG (but this number should be corrected due to the fact that high background is expected due to the high inclination of the orbit) and $\sim 40$ of them will be visible by all BDRG detectors 

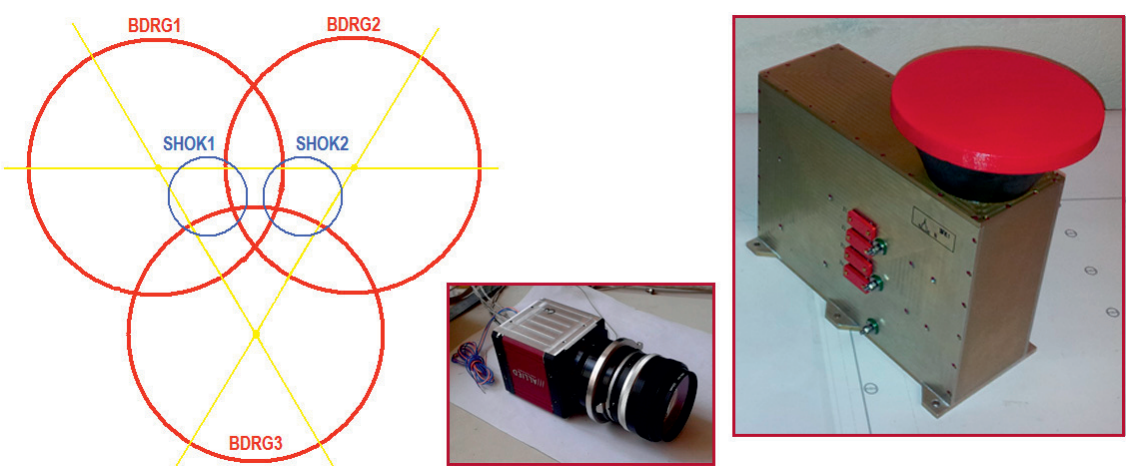

Fig. 6. Left: simplified FOV of SHOK and BDRG. Center: SHOK camera unit. Right: the SHOK camera unit embedded in its housing.

providing coordinate measurements. At most $\sim 10$ GRBs will appear in the FOV of SHOK cameras. For each trigger the light curve with $1 \mathrm{~ms}$ resolution for gamma rays and $200 \mathrm{~ms}$ for optical range will be obtained including history, providing new results in multiwavelength study of GRB prompt emission.

This work was partially supported by funds from Megagranta $\mathrm{N}^{\circ}$ 11.634.31.0076.

\section{References}

Gehrels, N., Ramirez-Ruiz, E., \& Fox, D.B., 2009, ARA\&A, 47, 567

Sadovnichii, V.A., Panasyuk, M.I., Amelushkin, A.M., et al., 2012, J. Cosmology, 18, 7999 
